\title{
Anti-Reflection Coating for Nitrogen-Vacancy Optical Measurements in Diamond
}

\section{Citation}

Yeung, T. K., D. Le Sage, L. M. Pham, P. L. Stanwix, and R. L. Walsworth. 2012. Anti-Reflection Coating for Nitrogen-Vacancy Optical Measurements in Diamond. Applied Physics Letters 100, no. $25: 251111$.

\section{Published Version}

doi:10.1063/1.4730401

\section{Permanent link}

http://nrs.harvard.edu/urn-3:HUL.InstRepos:11878775

\section{Terms of Use}

This article was downloaded from Harvard University's DASH repository, and is made available under the terms and conditions applicable to Other Posted Material, as set forth at http:// nrs.harvard.edu/urn-3:HUL.InstRepos:dash.current.terms-of-use\#LAA

\section{Share Your Story}

The Harvard community has made this article openly available.

Please share how this access benefits you. Submit a story.

\section{Accessibility}




\title{
Anti-reflection coating for nitrogen-vacancy optical measurements in diamond
}

\author{
T. K. Yeung, ${ }^{1,2}$ D. Le Sage, ${ }^{2,3}$ L. M. Pham, ${ }^{1}$ P. L. Stanwix, ${ }^{2,3}$ and R. L. Walsworth ${ }^{2,3}$ \\ ${ }^{1}$ School of Engineering and Applied Sciences, Harvard University, Cambridge, Massachusetts 02138, USA \\ ${ }^{2}$ Physics Department, Harvard University, Cambridge, Massachusetts 02138, USA \\ ${ }^{3}$ Harvard-Smithsonian Center for Astrophysics, Cambridge, Massachusetts 02138, USA
}

(Received 8 May 2012; accepted 5 June 2012; published online 22 June 2012)

\begin{abstract}
We realize anti-reflection (AR) coatings for optical excitation and fluorescence measurements of nitrogen-vacancy (NV) color centers in bulk diamond by depositing quarter-wavelength thick silica layers on the diamond surface. These AR coatings improve NV-diamond optical measurements by reducing optical reflection at the diamond-air interface from $\approx 17 \%$ to $\approx 2 \%$, which allows more effective NV optical excitation and more efficient detection of NV fluorescence. We also show that diamond AR coatings eliminate standing-wave interference patterns of excitation laser intensity within bulk diamond, and thereby greatly reduce spatial variations in NV fluorescence, which can degrade spatially resolved magnetic field sensing using NV centers. (c) 2012 American Institute of Physics. [http://dx.doi.org/10.1063/1.4730401]
\end{abstract}

The negatively charged nitrogen-vacancy (NV) color center in diamond has attracted considerable attention in recent years due to its many potential applications, including single-photon generation, ${ }^{1}$ quantum information processing, ${ }^{2,3}$ nanoscale and bulk magnetometry, ${ }^{4-11}$ and wide-field magnetic imaging. ${ }^{12,13}$ Many of these applications utilize the NV electronic spin state, which can be initialized and detected optically and coherently manipulated with microwave fields. ${ }^{14}$ The NV spin exhibits long coherence times $\left(T_{2} \approx 1 \mathrm{~ms}\right)$ in low-impurity bulk diamond samples at room temperature. ${ }^{15}$ Optical excitation of NV centers is typically driven with $532 \mathrm{~nm}$ laser light, and spin-state-dependent NV fluorescence is emitted in the $637-800 \mathrm{~nm}$ wavelength range. However, optical refraction and reflection at the bulk diamond surface can significantly limit NV spin-state excitation and readout fidelity when using a conventional microscope objective. A number of techniques have been employed to improve the NV fluorescence collection efficiency for bulk diamond, for example, by using a diamond solid immersion lens (SIL),${ }^{16,17}$ but losses from reflection at the diamond surface have thus far not been addressed. In particular, the diamond surface optical reflection coefficient can range from $5 \%$ for a diamond-oil interface (as used in oil-immersion microscope objectives) to $17 \%$ for a diamond-air interface (e.g., air objectives employed in cryogenic experiments ${ }^{3}$ or with SILs (Refs. 16 and 17)).

In this work, we report a straightforward method of producing an anti-reflection (AR) coating on a bulk diamond surface for improved NV excitation and fluorescence detection. The AR coating reduces the measured optical reflection coefficient at the diamond-air interface from $\approx 17 \%$ to $\approx 2 \%$, for light at both $532 \mathrm{~nm}$ and $632 \mathrm{~nm}$, consistent with our theoretical estimate. We also demonstrate that wide-field optical illumination of a planar diamond chip without an AR coating results in a characteristic spatial pattern of NV fluorescence that arises from interference between multiple reflections of the excitation laser beam within the diamond. This etalonlike or standing-wave interference pattern can degrade NV ensemble magnetometry ${ }^{5,9-11}$ and its use for sensitive measurements of temporally and spatially varying magnetic field patterns. ${ }^{12,13}$ We show that these optical interference patterns are largely eliminated by AR coating the diamond surface.

Diamonds with AR coatings are not widely available, and studies of diamond AR coatings in the scientific literature address resistance of the coating to hostile environments rather than optimal suppression of visible light reflections. ${ }^{18,19}$ In our work, we employed a simple and effective fabrication procedure using a single layer, quarterwavelength thick AR coating. The optimal refractive index of such a coating is given by

$$
n_{c}=\sqrt{n_{d} n_{i}},
$$

where $n_{d}$ is the refractive index of diamond and $n_{i}$ is the refractive index of the medium at the other interface of the coating. For a diamond-air interface, as studied in the experiments reported here, $n_{d}=2.42$ and $n_{i}=1.00$, yielding $n_{c}=1.56$. We chose silica $\left(\mathrm{SiO}_{2}\right)$ as the coating material because its refractive index $\left(n_{s}=1.46\right)$ is close to the desired value of $n_{c}$, and it is a low cost, robust, and easily accessible material.

We deposited silica AR coatings on two planar diamond samples via magnetron sputtering (AJA International ATC ORION 3 sputtering system) with a base pressure of $5 \times 10^{-8}$ Torr, an RF power of $148 \mathrm{~W}$, and a DC bias voltage of $142 \mathrm{~V}$. The typical deposition rate was $\sim 1 \mathrm{~nm} / \mathrm{min}$, as determined before each deposition with a trial run using a test sample, in which the thickness of the coating layer was measured using a surface profiler (Veeco) to within an uncertainty of a few $\mathrm{nm}$. The fraction of light reflected off the AR coated diamond surface at normal incidence is given by

$$
R_{A R}=R_{a s}+R_{s d}+2 \sqrt{R_{a s} R_{s d}} \cos \left[4 \pi t n_{s} / \lambda\right],
$$

where $R_{a s}$ and $R_{s d}$ are the Fresnel reflection coefficients at the air-silica and silica-diamond interfaces, respectively, $t$ is 


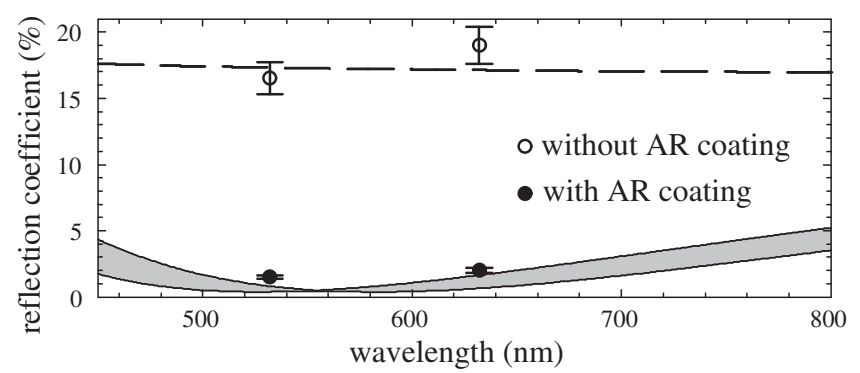

FIG. 1. Measured reflection coefficients for $532 \mathrm{~nm}$ and $632 \mathrm{~nm}$ light drop from $\approx 17 \%$ for a bare diamond surface (open circles) to $\approx 2 \%$ for an $\mathrm{AR}$ coated diamond surface (closed circles). Theoretical reflection coefficients, assuming near-normal incidence light, are indicated for a bare diamond surface (dashed line) and a diamond surface AR coated with a $95 \pm 5 \mathrm{~nm}$ thick silica layer (gray shaded region), assuming air is the surrounding medium.

the thickness of the silica layer, $n_{s}$ is the refractive index of silica, and $\lambda$ is the wavelength of the light. The final silica thickness measured with the surface profiler $(t=95 \pm 5 \mathrm{~nm})$ closely matched the value chosen to minimize the reflection of both $532 \mathrm{~nm}$ excitation light and red NV fluorescence. The silica layer robustly adhered to the diamonds but could be removed using a $0.3 \mu \mathrm{m}$ grit fiber-polishing film, if necessary.

For our optical reflection measurements, we AR coated half of one surface of a polished diamond plate that was $9 \mathrm{~mm} \times 8 \mathrm{~mm} \times 1.2 \mathrm{~mm}$ in size. This plate was composed of six [100]-oriented synthetic diamonds produced via chemical vapor deposition (CVD), which were bonded together and then polished. We measured the fraction of light reflected from each half of the diamond surface, with and without AR coating, using collimated $532 \mathrm{~nm}$ and $632 \mathrm{~nm}$ wavelength laser beams. By tilting the beams slightly away from normal incidence to the diamond, it was possible to distinguish between the beams reflected off of the front and back surfaces of the diamond and, therefore, to measure the reflection coefficient of a single surface. In Fig. 1, we compare these measurements against theoretical estimates made using the Fresnel equations, Eq. (2), and the wavelength-dependent refractive indices of silica and diamond. ${ }^{20}$ Our measurements show that for both wavelengths, the reflection coefficient drops from $\approx 17 \%$ for a bare diamond surface to $\approx 2 \%$ for an AR coated diamond surface, in reasonable agreement with our theoretical estimates.

In addition to degrading NV excitation and fluorescence detection, reflections of the diamond interfaces can also cause significant spatial variations in the excitation light intensity within the diamond. This effect is caused by interference between incident and reflected laser light within the polished diamond slab, which behaves as a low-finesse ( $F \simeq 1$ ) etalon. This interference effect is particularly evident in wide-field illumination of ensembles of NV centers, as employed for demonstrations of vector magnetic field imaging using a charge-coupled device (CCD) detector. ${ }^{12}$ Such techniques provide spatiotemporally resolved sensitive vector magnetometry within a wide field-of-view for studying magnetic field sources near the diamond interface. Because the magnetic field signal is extracted from the NV fluorescence rate, spatial variations in the excitation light intensity can cause systematic errors in spatially resolved NV magnetometry and should therefore be minimized.
To demonstrate this interference effect, we used a widefield fluorescence microscope to image spatial variations of NV fluorescence onto a CCD [Fig. 2(a)]. We employed a different diamond sample than for the reflection measurements described above: a $300 \mu \mathrm{m}$ thick diamond substrate produced via CVD with very low concentrations of substitutional nitrogen atoms (N), NV centers, and other impurities, which was implanted with nitrogen ions and annealed, producing a very thin $(\approx 10 \mathrm{~nm}$ thick) layer of $\mathrm{NV}$ centers near one surface. The $532 \mathrm{~nm}$ excitation laser beam (Gaussian intensity profile) was focused in front of the diamond sample (focused beam diameter $\approx 2.8 \mu \mathrm{m}$ ) in order to illuminate the thin layer of NV centers in $\mathrm{a} \approx 100 \mu \mathrm{m}$ wide region [Fig. 2(b)]. We modeled the distribution of excitation light within the diamond sample resulting from this incident illumination profile and Fresnel reflections at the sample surfaces. Considering the incident light and the effect of two internal reflections within the diamond sample, we calculate the intensity distribution of excitation light at the NV layer to be

$$
I(r)=I_{0}\left|E\left(r, z_{1}\right)+r^{2} E\left(r, z_{2}\right)+r^{4} E\left(r, z_{3}\right)\right|^{2},
$$

where $I_{0}$ is a constant scaling factor, $E(r, z)$ is the electric field amplitude of a Gaussian beam, $r=\sqrt{R_{a d}}$ is the amplitude reflectivity of the air-diamond interface, and $z_{1}, z_{2}$, and $z_{3}$ are the longitudinal distances of the NV layer from the waist of the incident beam and the second and third reflected beams, respectively. This model of the intensity distribution [Figs. 2(c) and 2(e)] agrees well with the measured NV fluorescence distribution recorded by the CCD [Figs. 2(d) and 2(f)]. This agreement is expected, since the NV fluorescence rate scales linearly with the excitation intensity in the range of intensities used here.

We next demonstrated that such spatial variations in NV fluorescence can be greatly reduced with AR coatings on one or more of the diamond surfaces. We used a [100]-oriented, CVD-grown, $5 \mathrm{~mm} \times 5 \mathrm{~mm} \times 0.2 \mathrm{~mm}$ diamond sample with a uniform density of $\mathrm{NV}$ centers $\left(\approx 10^{14} \mathrm{~cm}^{-3}\right)$. The diamond's surfaces were divided into four $2.5 \mathrm{~mm} \times 2.5 \mathrm{~mm}$ regions, with either no AR coating, a coating on a single side, or coatings on both sides. This diamond was AR coated at the same time as the diamond used for the reflection measurements shown in Fig. 1. We imaged transverse spatial patterns of NV fluorescence using the CCD-based microscope depicted in Fig. 2(a). When the uncoated region of the diamond sample was illuminated with $532 \mathrm{~nm}$ excitation light, the observed transverse NV fluorescence distribution exhibited a characteristic interference pattern [Fig. 3(a)]. Because of the uniform distribution of NV centers in this diamond, the interference features are not as sharp as in Fig. 2(d), for which the NV centers were constrained to a thin layer near the diamond surface. When we instead illuminated the region of the uniform-NV-distribution diamond with AR coatings on both sides, the observed NV fluorescence interference pattern was greatly reduced [Fig. 3(b)]. We found that having an AR coating on just one of the diamond surfaces also greatly reduced this interference effect, thus eliminating the need for an additional $100 \mathrm{~nm}$ separation between the NV centers and the magnetic sample being probed. By comparing NV fluorescence signals from regions with and 
(a)

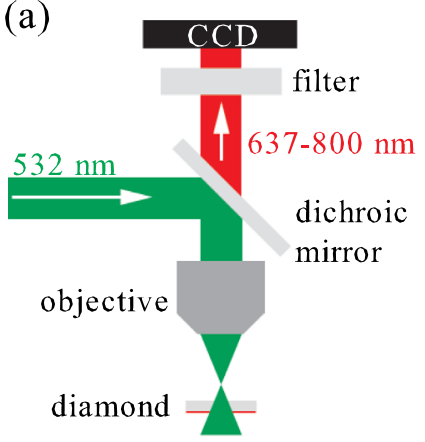

(c)

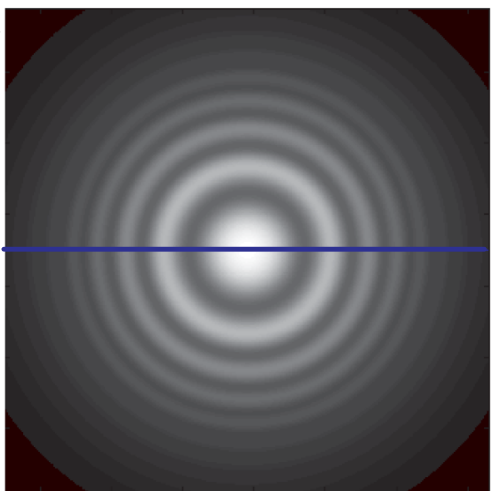

(e)

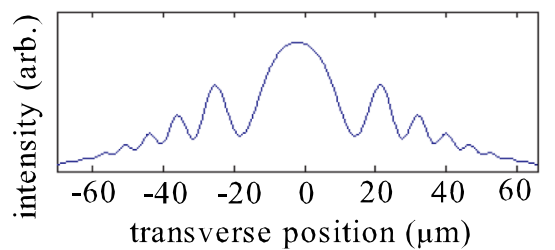

(b)
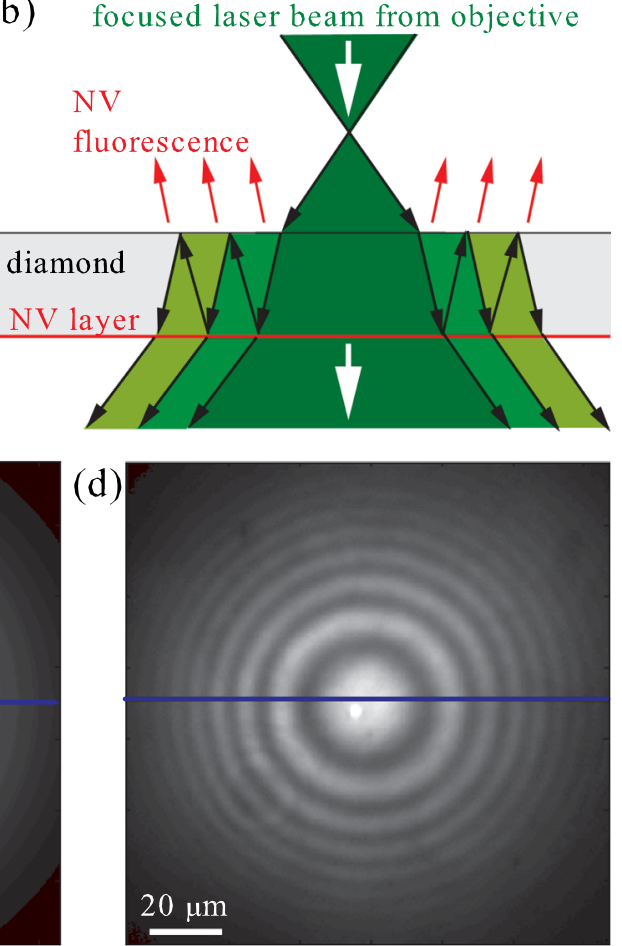

(f)

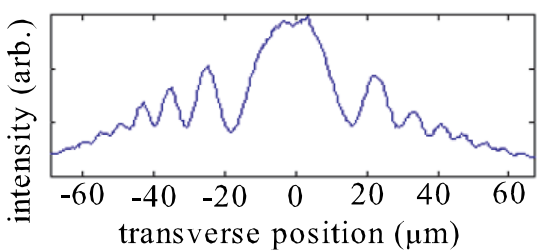

FIG. 2. (a) Setup used to capture wide-field NV fluorescence images using a CCD detector. (b) Reflections of the focused $532 \mathrm{~nm}$ laser beam from uncoated diamond surfaces interfere to create transverse spatial intensity variations of the excitation light at a thin layer of $\mathrm{NV}$ centers at one diamond surface, which induce corresponding transverse spatial variations in NV fluorescence. (c) Calculated transverse spatial variation of NV fluorescence, based upon measured properties of the focused $532 \mathrm{~nm}$ laser beam (incident excitation light), density of NV centers in the thin surface layer, and diamond thickness. (d) Corresponding measured transverse pattern of NV fluorescence intensity recorded on CCD. (e) and (f) Intensity as a function of transverse position along blue lines in (c) and (d), respectively. without an AR coating on the side of the diamond facing the objective, we estimated that the AR coating improved NV fluorescence transmission by $\approx 16 \%$, which was consistent with the expected improvement shown in Fig. 1. We did not see any evidence of additional fluorescence originating from the silica coating as $532 \mathrm{~nm}$ excitation light passed through it.

In summary, we have demonstrated a simple, effective procedure for producing an $\mathrm{AR}$ coating for a diamond-air interface, increasing both the NV center excitation and fluo-

(a)
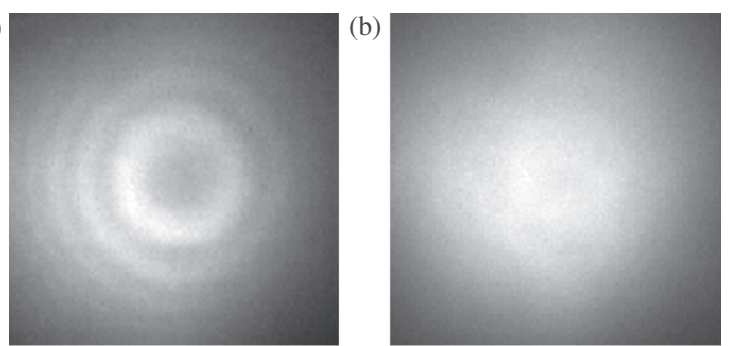

FIG. 3. (a) Observed NV fluorescence distribution in the uncoated region of a diamond sample with uniform distribution of NV centers, exhibiting a characteristic interference pattern due to reflections of $532 \mathrm{~nm}$ excitation light at the diamond surfaces. (b) Observed NV fluorescence interference pattern is largely suppressed in the region of the diamond with AR coatings on both sides. Reproduced with permission from L. M. Pham et al., New J. Phys. 13, 045021 (2011). Copyright @ 2011 Institute of Physics Publishing Ltd. rescence detection efficiencies. This technique may be easily extended to AR coatings appropriate for other fluorescent defect centers in diamond by varying the AR coating thickness to match the wavelength of the corresponding fluorescence band. Similarly, an AR coating appropriate for a diamond-oil interface may be created by sputtering a material with a refractive index close to 1.9 , such as $\mathrm{Yb}_{2} \mathrm{O}_{3}$ and $\mathrm{Y}_{2} \mathrm{O}_{3}$ coatings that have previously been used with diamond. ${ }^{18}$ We have shown that AR coatings allow uniform optical excitation of NV centers in diamond within a large field of view, which will be important for optimizing the sensitivity of bulk NV-diamond magnetometry ${ }^{5,9-11}$ and magnetic field imaging using ensembles of NV centers. ${ }^{12,13}$ Such an AR coating could also reduce scattering losses in absorptionbased NV ensemble measurements, ${ }^{21}$ improve NV spin state measurement fidelity for magnetometry ${ }^{4-6,9-11}$ and quantum information applications, ${ }^{3}$ and improve the efficiency of NV single photon sources. ${ }^{16,17}$

We gratefully acknowledge the provision of diamond samples by Apollo Diamond and Element Six. This work was supported by NIST, NSF, and DARPA (QuEST and QuASAR programs).

${ }^{1}$ C. Kurtsiefer, S. Mayer, P. Zarda, and H. Weinfurter, Phys. Rev. Lett. 85, 290 (2000).

${ }^{2}$ M. V. G. Dutt, L. Childress, L. Jiang, E. Togan, J. Maze, F. Jelezko, A. S. Zibrov, P. R. Hemmer, and M. D. Lukin, Science 316, 1312 (2007). 
${ }^{3}$ E. Togan, Y. Chu, A. S. Trifonov, L. Jiang, J. Maze, L. Childress, M. V. G. Dutt, A. S. Sorensen, P. R. Hemmer, A. S. Zibrov, and M. D. Lukin, Nature 466, 730 (2010).

${ }^{4}$ J. R. Maze, P. L. Stanwix, J. S. Hodges, S. Hong, J. M. Taylor, P. Cappellaro, L. Jiang, M. V. G. Dutt, E. Togan, A. S. Zibrov, A. Yacoby, R. L. Walsworth, and M. D. Lukin, Nature 455, 644 (2008).

${ }^{5}$ J. Taylor, P. Cappellaro, L. Childress, L. Jiang, D. Budker, P. R. Hemmer, A. Yacoby, R. Walsworth, and M. D. Lukin, Nat. Phys. 4, 810 (2008).

${ }^{6}$ P. C. Maurer, J. R. Maze, P. L. Stanwix, L. Jiang, A. V. Gorshkov, A. A. Zibrov, B. Harke, J. S. Hodges, A. S. Zibrov, A. Yacoby, D. Twitchen, S. W. Hell, R. L. Walsworth, and M. D. Lukin, Nat. Phys. 6, 912 (2010).

${ }^{7}$ M. S. Grinolds, P. Maletinsky, S. Hong, M. D. Lukin, R. L. Walsworth, and A. Yacoby, Nat. Phys. 7, 687 (2011).

${ }^{8}$ P. Maletinsky, S. Hong, M. S. Grinolds, B. Hausmann, M. D. Lukin, R. L. Walsworth, M. Loncar, and A. Yacoby, Nat. Nanotechnol. 7, 320 (2012).

${ }^{9}$ D. Le Sage, L. M. Pham, N. Bar-Gill, C. Belthangady, M. D. Lukin, A. Yacoby, and R. L. Walsworth, Phys. Rev. B 85, 121202 (2012).

${ }^{10}$ P. L. Stanwix, L. M. Pham, J. R. Maze, D. Le Sage, T. K. Yeung, P. Cappellaro, P. R. Hemmer, A. Yacoby, M. D. Lukin, and R. L. Walsworth, Phys. Rev. B 82, 201201 (2010).

${ }^{11}$ N. Bar-Gill, L. M. Pham, C. Belthangady, D. Le Sage, P. Cappellaro, J. R. Maze, M. D. Lukin, A. Yacoby, and R. Walsworth, Nat. Commun. 3, 858 (2012).
${ }^{12}$ L. M. Pham, D. Le Sage, P. L. Stanwix, T. K. Yeung, D. Glenn, A. Trifonov, P. Cappellaro, P. R. Hemmer, M. D. Lukin, H. Park, A. Yacoby, and R. L. Walsworth, New J. Phys. 13, 045021 (2011).

${ }^{13}$ S. Steinert, F. Dolde, P. Neumann, A. Aird, B. Naydenov, G. Balasubramanian, F. Jelezko, and J. Wrachtrup, Rev. Sci. Instrum. 81, 043705 (2010).

${ }^{14}$ F. Jelezko, T. Gaebel, I. Popa, A. Gruber, and J. Wrachtrup, Phys. Rev. Lett. 92, 076401 (2004).

${ }^{15}$ G. Balasubramanian, P. Neumann, D. Twitchen, M. Markham, R. Kolesov, N. Mizuochi, J. Isoya, J. Achard, J. Beck, J. Tissler, V. Jacques, P. R. Hemmer, F. Jelezko, and J. Wrachtrup, Nature Mater. 8, 383 (2009).

${ }^{16}$ P. Siyushev, F. Kaiser, V. Jacques, I. Gerhardt, S. Bischof, H. Fedder, J. Dodson, M. Markham, D. Twitchen, F. Jelezko, and J. Wrachtrup, Appl. Phys. Lett. 97, 241902 (2010).

${ }^{17}$ J. P. Hadden, J. P. Harrison, A. C. Stanley-Clarke, L. Marseglia, Y.-L. D. Ho, B. R. Patton, J. L. O'Brien, and J. G. Rarity, Appl. Phys. Lett. 97, 241901 (2010).

${ }^{18}$ T. P. Mollart and K. L. Lewis, Diamond Relat. Mater. 10, 536 (2001).

${ }^{19}$ Y. Tzeng, Diamond Films Technol. 1, 31 (1991).

${ }^{20}$ M. Bass, G. Li, and E. W. Van Stryland, Handbook of Optics (McGrawHill, 2009), Vol. 4.

${ }^{21}$ V. M. Acosta, E. Bauch, A. Jarmola, L. J. Zipp, M. P. Ledbetter, and D. Budker, Appl. Phys. Lett. 97, 174104 (2010). 